UNIL-IPT-00-18

IC/2000/133

hep-th/0008087

August 2000

\title{
On some new warped brane world solutions in higher dimensions
}

\author{
Seif Randjbar-Daemi ${ }^{a}$ and Mikhail Shaposhnikov ${ }^{b}$ \\ ${ }^{a}$ International Center for Theoretical Physics, Trieste, Italy \\ ${ }^{b}$ Institute of Theoretical Physics, University of Lausanne, \\ CH-1015 Lausanne, Switzerland
}

\begin{abstract}
We present new solutions of higher dimensional Einstein's equations with a cosmological constant that localize gravity on branes which are transverse to Ricci-flat manifolds or to homogeneous spaces with topologically non-trivial solutions of gauge field equations. These solutions are relevant for the localization of chiral fermions on a brane.
\end{abstract}


Although the non-compact internal spaces [1]- [4] have been considered in the past, the recent interest, inspired by ideas from superstring theory, emphasizes the notion that our largescale universe may be regarded as a brane embedded in a higher-dimensional manifold. The gravity may be localized on a brane by the Randall-Sundrum mechanism [5], while the observed particles are attached to a brane because of dynamics of string theory [6] or by field-theoretic effects [1], [7]-[10]. It thus becomes relevant to look for general solutions of the $D$-dimensional field equations. It is expected that a richer geometrical and topological structure of the subspaces spanned by extra dimensions will lead to more interesting physical consequences on the brane world. A number of warped solutions have already been found in [2, 3], [11]-19].

In this note, we consider a $D=D_{1}+D_{2}+1$-dimensional system of gravity - Yang-Mills system and look for warped solutions of the form

$$
\mathrm{d} s^{2}=\mathrm{e}^{A(r)} \eta_{\mu \nu} \mathrm{d} x^{\mu} \mathrm{d} x^{\nu}+\mathrm{e}^{B(r)} g_{m n}(y) \mathrm{d} y^{m} \mathrm{~d} y^{n}+\mathrm{d} r^{2}
$$

where $x^{0}, x^{1}, \ldots, x^{D_{1}-1}$ cover the brane world $M_{D_{1}}$ and $y^{1}, \ldots, y^{D_{2}}$ cover an internal space $K$ with a metric $g_{m n}(y)$. The metric with $D_{2}=0$ and $A=c|r|, c<0$ is the Randall-Sundrum case [5], the one with $D_{2}=1$ and $A=B=c|r|, c<0$ is the case of local string [13], $D_{2}=1$ and $A=c|r|, c<0, B=$ const corresponds to a global string [12]. When $D_{2} \geq 2$ the approximate solutions with scalar fields [14 or exact bulk solutions with $p$-form fields [16] have all assumed that $K=S^{D_{2}}$. In this note we shall consider cases where $K$ is not restricted to being a sphere. These general cases become important if we want to localize chiral fermions to the brane world $M_{D_{1}}$ [10].

In the presence of Yang-Mills fields we shall assume that $K$ is a symmetric homogeneous space $G / H$ with a $G$-invariant metric $g_{m n}(y)$ defined on it. We shall also assume that the gauge group has a non-trivial intersection with $H$. For example, for $G / H=S^{4}$ it is sufficient to assume that the gauge group contains an $\mathrm{SU}(2)$ subgroup. On such spaces one can construct $G$-invariant solutions to the Yang-Mills equations [20], giving

$$
\vec{F}_{m p} \cdot \vec{F}_{n}^{p}=\frac{\mathrm{e}^{-B}}{D_{2}} F^{2} g_{m n}
$$

where, by virtue of $G$-invariance,

$$
F^{2} \equiv g^{m p} g^{n q} \vec{F}_{m n} \cdot \vec{F}_{p q}
$$

must be a constant. The non-trivial equations to be solved can then be put into the form

$$
\begin{gathered}
A^{\prime \prime}+\frac{D_{1}}{2} A^{\prime 2}+\frac{D_{2}}{2} A^{\prime} B^{\prime}=\frac{4 \kappa^{2}}{D-2}\left(-\Lambda+\frac{F^{2}}{4 g^{2}} \mathrm{e}^{-2 B}\right), \\
B^{\prime \prime}+\frac{D_{2}}{2} B^{\prime 2}+\frac{D_{1}}{2} A^{\prime} B^{\prime}=\frac{4 \kappa^{2}}{D-2}\left(-\Lambda-\frac{2 D-D_{1}-4}{D_{2}} \frac{F^{2}}{4 g^{2}} \mathrm{e}^{-2 B}\right)+k \mathrm{e}^{-B}, \\
D_{1} A^{\prime \prime}+D_{2} B^{\prime \prime}+\frac{D_{1}}{2} A^{\prime 2}+\frac{D_{2}}{2} B^{\prime 2}=\frac{4 \kappa^{2}}{D-2}\left(-\Lambda+\frac{F^{2}}{4 g^{2}} \mathrm{e}^{-2 B}\right),
\end{gathered}
$$


where $\Lambda$ and $\kappa^{2}$ are $D$-dimensional cosmological and Newtonian constants respectively, $g$ is the gauge coupling. Also the constant $k$ in eq. (5) is the curvature scalar of $K$ defined by $R_{m n}=k g_{m n}$. It can be shown that only two out of the three equations above are independent. It should also be noted that these equations are valid in the bulk, the brane core region around $r=0$ should be treated in the same way as in [13, 16], which provides a relationship between different tensions on the brane and the values of $A^{\prime}$ and $B^{\prime}$ near the origin.

At this point it is convenient to consider the cases $k=0$ and $k \neq 0$ separately.

(i) $k=0$. In this case $K$ is a Ricci flat manifold. Well known examples are tori and Calabi-Yau manifolds. Setting $F^{2}=0$, one can find all solutions of eqs. (4-6), generalizing the solutions found in [2, 3, 16] for $D_{1}=4, D=6$, where the $k$-term is trivially absent. The general result is:

$$
A(r)=\operatorname{alog}\left[z^{\prime}(r)\right]+b \log [z(r)], \quad B(r)=c \log \left[z^{\prime}(r)\right]+d \log [z(r)]
$$

where

$$
\begin{aligned}
& a=\frac{2}{D-1}\left(1-\sqrt{\frac{D_{2}(D-2)}{D_{1}}}\right), \quad b=\frac{2}{D-1}\left(1+\sqrt{\frac{D_{2}(D-2)}{D_{1}}}\right), \\
& c=\frac{2}{D-1}\left(1+\sqrt{\frac{D_{1}(D-2)}{D_{2}}}\right), \quad d=\frac{2}{D-1}\left(1-\sqrt{\frac{D_{1}(D-2)}{D_{2}}}\right),
\end{aligned}
$$

and

$$
z(r)=\operatorname{Re}\left(\alpha e^{\gamma r}+\beta e^{-\gamma r}\right), \quad \gamma=\sqrt{-\frac{(D-1) \kappa^{2} \Lambda}{2(D-2)}},
$$

where $\alpha$ and $\beta$ are arbitrary constants.

It is easy to verify that for a negative cosmological constant there is a simple solution with $A=B=-\gamma r$, where $\gamma$ is not given by $(10)$ but $\gamma=\sqrt{-\frac{8 \kappa^{2} \Lambda}{(D-1)(D-2)}}$. As in the standard RS solution, we can extend the range of $r$ to $(-\infty,+\infty)$ and write the solution as $A=B=-\gamma|r|$. In this case $A^{\prime \prime}=B^{\prime \prime}=-2 \gamma \delta(r)$. The field equations in the core region will then relate the tension of the brane to $\gamma$, along the lines discussed in [5, 13, 16]. At infinity, $r \rightarrow \infty$, the square of the curvature tensor is singular, as follows from the general expression, given in [3]. The only exception is when the internal space is flat, for example, it can be a torus. In this case the soltuion is non singular even as $r \rightarrow \infty$. For the general solution to make physical sense the singularity should be smoothen by, say, string theory effects.

(ii) $k \neq 0$. In this case the $G$-invariant solutions of Yang-Mills equations can be constructed as in [21]. One can have a regular solution in the bulk with the structure $A \mathrm{~d} S_{D_{1}+1} \times G / H$ with $A=-c r$ and $B$ a constant, similar to the one considered in [16] for a compactification on a 
monopole for $D_{2}=2$. One can consider two possibilities. In the first case we can treat $r$ as a radial co-ordinate, varying fom zero to infinity. Then this solution is singular at $r=0$ and requires the addition of a $G$ invariant brane, residing at this point. The tensions on the brane must then be fine tuned accordingly. In the second case $r$ may vary from $-\infty$ to $+\infty$. As the Einstein equations are symmetric with respect to the transformation $r \rightarrow-r$, a solution, leading to the localization of gravity, looks like $A=-c|r|$ and requires the presence of a brane as well.

An interesting question arises whether one can construct a braneless solution that is regular at $r=0$ and which approaches the bulk solution just described for large values of $r$. Arguing along the lines of ref. [3], this seems to be impossible, at least if $-\infty<r<\infty$, when the $Z_{2}$ symmetry is imposed. To this end it is convenient to rewrite eqs. (44 6) as a single equation for $B$ only,

$$
\begin{gathered}
B^{\prime \prime}=\frac{1}{2(D-2)\left(D_{1}-1\right) D_{2} e^{2 B}}\left\{(D-2) D_{2}^{2} B^{\prime 2} e^{2 B}+\right. \\
\left.+2\left(D_{1}-1\right)\left(\left(4-2 D+D_{1}\right) \frac{\kappa^{2} F^{2}}{g^{2}}-D_{2}\left(2 k-D k+4 e^{B} \kappa^{2} \Lambda\right) e^{B}\right) \pm S\right\}
\end{gathered}
$$

where

$$
\begin{gathered}
S=D_{2} \sqrt{D_{1}(D-2)} B^{\prime} e^{B}\left\{D_{2}(D-2)^{2} B^{\prime 2} e^{2 B}-\right. \\
\left.2\left(D_{1}-1\right)\left(\left(2 D-2 D_{1}-3\right) \frac{\kappa^{2} F^{2}}{g^{2}}+4\left(D_{1}-1\right) e^{2 B} \kappa^{2} \Lambda+D_{2}\left((2-D) k+4 e^{B} \kappa^{2} \Lambda\right) e^{B}\right)\right\}^{\frac{1}{2}}
\end{gathered}
$$

Now, this equation describes a motion of a particle in a potential $V(B)$ which is obtained by setting $B^{\prime}=0$ in eq. (12) and integrating with respect to $B$.

$$
V(B)=k e^{-B}-\frac{1}{(D-2)}\left\{\frac{1}{2 D_{2}}\left(2 D-D_{1}-4\right) \frac{\kappa^{2} F^{2}}{g^{2}} e^{-2 B}-4 \kappa^{2} \Lambda B\right\}
$$

with quite complicated friction force. The regularity of solution at $r=0$ requires $B^{\prime}(0)=0$, while $B \rightarrow$ const at infinity. In a mechanical analogy the particle should start moving with zero velocity and reach the maximum of the potential at "time" $r \rightarrow \infty$. This is only possible if the potential (13) has two extrema. This requirement leads to a certain constraints on the parameters $\frac{\kappa^{2} F^{2}}{k g^{2}}, \frac{\kappa^{2} \Lambda}{k}$ and on the dimensionalities $D_{1}$ and $D_{2}$. The latter happen to be not consistent with reality of the quantity $S$ defined in eq. (12) at the maximum of the potential.

Both types of solutions (i) and (ii) can be used to obtain localized chiral fermions in $M_{D_{1}}$. As argued in [10] for the case $K=K_{3}$ and $D=9$, the index of the Dirac operator $\nabla$ acting in the transverse space is two, which implies the existence of two families of chiral fermions in $M_{4}$. Alternatively, if we choose $K=S^{4}$ and $\vec{F}$ an $S U(2)$ instanton configuration on $S^{4}$ then 
we obtain $\frac{2}{3} t(t+1)(2 t+1)$ generations of chiral fermions on $M_{4}$, where $t$ is the $S U(2)$ spin of the fermions [21].

In conclusion, we found new solutions that localize gravity on a brane with non-trivial transverse spaces and gauge instanton backgrounds. It remains to be seen if a realistic theory incorporating fields of the Standard Model can be constructed on these solutions.

We wish to thank T. Gherghetta for helpful discussions. S. R.-D. is thankful to IPT-UNIL for hospitality. This work was supported by the FNRS, contract no. 21-55560.98.

\section{References}

[1] V. A. Rubakov and M. E. Shaposhnikov, Phys. Lett. B125 (1983) 136.

[2] V. A. Rubakov and M. E. Shaposhnikov, Phys. Lett. B125 (1983) 139.

[3] S. Randjbar-Daemi and C. Wetterich, Phys. Lett. B166 (1986) 65.

[4] K. Akama, in Proceedings of the Symposium on Gauge Theory and Gravitation, Nara, Japan, eds. K. Kikkawa, N. Nakanishi and H. Nariai (Springer-Verlag, 1983);

M. Visser, Phys. Lett. B159 (1985) 22.

[5] L. Randall and R. Sundrum, Phys. Rev. Lett. 83 (1999) 4690.

[6] J. Polchinski, Phys. Rev. Lett. 75 (1995) 4724.

[7] G. Dvali and M. Shifman, Nucl. Phys. B504 (1997) 127, Phys. Lett. B396 (1997) 64

[8] B. Bajc and G. Gabadadze, Phys. Lett. B474 (2000) 282.

[9] I. Oda, hep-th/0006203.

[10] S. Randjbar-Daemi and M. Shaposhnikov, hep-th/0008079

[11] A. G. Cohen and D. B. Kaplan, Phys. Lett. B470 (1999) 52

[12] R. Gregory, Phys. Rev. Lett. 84 (2000) 2564.

[13] T. Gherghetta and M. Shaposhnikov, Phys. Rev. Lett. 85 (2000) 240.

[14] I. Olasagasti and A. Vilenkin, hep-th/0003300.

[15] G. Dvali, hep-th/0004057.

[16] T. Gherghetta, E. Roessl and M. Shaposhnikov, hep-th/0006251.

[17] J. Chen, M. A. Luty and E. Ponton, hep-th/0003067.

[18] A. Chodos, E. Poppitz and D. Tsimpis, hep-th/0006093. 
[19] C. Csaki, J. Erlich, T. J. Hollowood and Y. Shirman, hep-th/0001033.

[20] S. Randjbar-Daemi and R. Percacci, Phys. Lett. B117 (1982) 41.

[21] S. Randjbar-Daemi, A. Salam and J. Strathdee, Phys. Lett. B132 (1983) 56; Nucl. Phys. B214 (1983) 491. 